\title{
STRONG TYPE ENDPOINT BOUNDS FOR ANALYTIC FAMILIES OF FRACTIONAL INTEGRALS
}

\author{
LOUKAS GRAFAKOS
}

(Communicated by J. Marshall Ash)

\begin{abstract}
In $\mathbb{R}^{2}$ we consider an analytic family of fractional integrals, whose convolution kernel is obtained by taking some transverse derivatives of arclength measure on the parabola $\left(t, t^{2}\right)$ multiplied by $|t|^{\gamma}$ and doing so in a homogeneous way. We determine the exact range of $p, q$ for which the analytic family maps $L^{p}$ to $L^{q}$. We also resolve a similar issue on the Heisenberg group.
\end{abstract}

\section{INTRODUCTION}

In $\mathbb{R}^{2}$ consider the following family of operators:

$$
S^{\gamma}(f)(x)=\int_{-\infty}^{\infty} f\left(x_{1}-t, x_{2}-t^{2}\right)|t|^{\gamma} \frac{d t}{t} \quad \text { where } 0 \leq \gamma \leq 1,
$$

where the integral in (1.1) is interpreted in the principal value sense when $\gamma=0$. For $\gamma>0$, the operators $S^{\gamma}$ are called fractional integrals along the parabola $\left(t, t^{2}\right)$ and have been studied by Ricci and Stein [RS] and Christ [C2], who determined the range of $(1 / p, 1 / q, \gamma)$ for which $S^{\gamma}$ maps $L^{p}$ to $L^{q}$. By homogeneity such a boundedness result can happen only when $1 / p-1 / q=$ $\operatorname{Re} \gamma / 3$. In $\mathbb{R}^{2}$, let $\Delta$ be the closed triangle with vertices $(0,0),(1,1)$, and $(2 / 3,1 / 3)$, and let $\Gamma$ be the part of $\Delta$ that does not contain the diagonal. [RS] proved $L^{p} \rightarrow L^{q}$ boundedness for $S^{\gamma}$ when $(1 / p, 1 / q)$ lie in $\Gamma$ minus the piece of the boundary $\{(1 / p, 1 / q): q=2 p$ and $2<p<\infty\}$ union its reflection across the line $1 / q=1-1 / p$. [C2] proved $L^{p} \rightarrow L^{q}$ boundedness for the remaining boundary points of $\Gamma$ that do not lie on the diagonal $p=q$. (When $\gamma=0, S^{0}$ is the Hilbert transform along the parabola and it is bounded on the diagonal for $1<p<\infty$. See [SWA] for details.) Furthermore it is known from [RS] that no positive result for $S^{\gamma}$ holds outside $\Gamma$ when $\gamma>0$.

We prove a similar result as in [C2, RS], for an analytic family of fractional integrals along the parabola $S_{z}^{\gamma}$, in which the operators $S^{\gamma}$ can be embedded. The convolution kernel of $S_{z}^{\gamma}$ is obtained by taking $-z-1$ transverse derivatives of arclength measure on the parabola, multiplied by $|t|^{\gamma}$, and doing so in a homogeneous way. The analytic family $S_{z}^{\gamma}$ is defined in such a way as to satisfy $S_{-1}^{\gamma}=S^{\gamma}$.

Received by the editors December 6, 1989 and, in revised form, April 9, 1991.

1991 Mathematics Subject Classification. Primary 43A80. 
We now give a precise definition of $S_{z}^{\gamma}$. Fix an even nonnegative function $\psi \in C_{0}^{\infty}(\mathbb{R})$ supported in $[-1,1]$ and equal to 1 on $[-1 / 2,1 / 2]$. Also fix $\gamma \in \mathbb{C}$ with $\operatorname{Re} \gamma \geq 0$. For $f$ smooth with compact support in $\mathbb{R}^{2}$, we define

$$
\left(S_{z}^{\gamma} f\right)(x)=\int_{-\infty}^{\infty} \int 2 \Gamma\left(\frac{z+1}{2}\right)^{-1}|u-1|^{z} \psi(u-1) f\left(x_{1}-t, x_{2}-u t^{2}\right) d u|t|^{\gamma} \frac{d t}{t},
$$

where the outer integral is to be interpreted in the principal value sense, when $\operatorname{Re} \gamma=0$. $S_{z}^{\gamma}$ is initially defined for $\operatorname{Re} z>-1$. By analytic continuation, see [GS], the definition of $S_{z}^{\gamma}$ can be extended for all $z$ complex. Because of the $\Gamma$ function normalization we get that $S_{-1}^{\gamma}=S^{\gamma}$, for all $\gamma$ with $\operatorname{Re} \gamma \geq 0$. $S_{z}^{\gamma}$ depends analytically on both $\gamma$ and $z$ and, therefore, is a double analytic family of operators with parameters $(z, \gamma) \in \mathbb{C} \times \mathbb{C}_{+}$, where by $\mathbb{C}_{+}$we denote the set of all complex numbers with nonnegative real part.

Our first result describes the exact range of $(1 / p, 1 / q, z, \gamma)$ for which $S_{z}^{\gamma}$ maps $L^{p}\left(\mathbb{R}^{2}\right)$ to $L^{q}\left(\mathbb{R}^{2}\right)$ when $\gamma>0$. Since such a boundedness result can only hold when $\operatorname{Re} \gamma / 3=1 / p-1 / q$, it is enough to describe the possible range of $p, q$, and $z$. Our first theorem is the following:

Theorem 1. For $\operatorname{Re} \gamma>0$, the analytic family of fractional integrals $S_{z}^{\gamma}$ maps $L^{p}$ to $L^{q}$ if and only if $(1 / p, 1 / q, \operatorname{Re} z)$ lies on or vertically above the interiors of the faces $\mathrm{BCD}$ and $\mathrm{ABD}$ union the edge $\mathrm{BD}-\{\mathrm{B}\}$ of the tetrahedron $\mathrm{ABCD}$ with vertices $\mathrm{A}=(0,0,-1), \mathrm{B}=(1 / 2,1 / 2,-3 / 2), \mathrm{C}=(1,1,-1)$, and $\mathrm{D}=(1,0,0) .($ See Figure 1.$)$

We use Theorem 1 in [C2] to treat the main part of the kernel of $S_{z}^{\gamma}$ for a certain range of $z$ 's but we do not follow the method of Christ's proof since the positivity of the kernel of $S^{\gamma}$ was essential in the treatment of this operator in his work. Throughout this paper $C_{z, \gamma}, c_{z, \gamma}$ will denote constants that grow

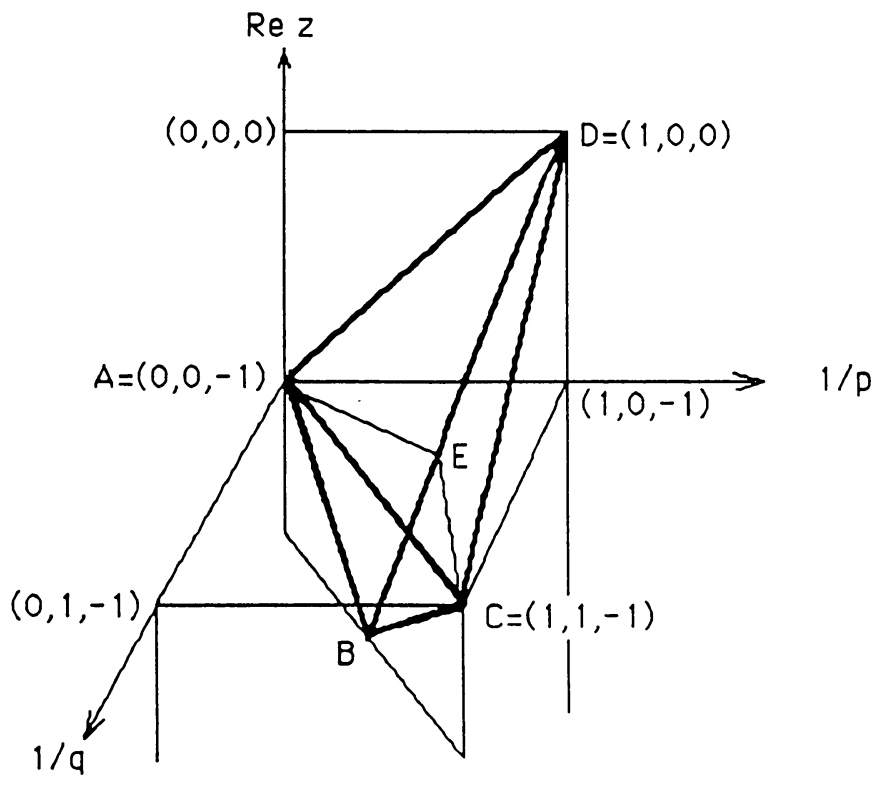

Figure 1. $\mathrm{B}=(1 / 2,1 / 2,-3 / 2), \mathrm{E}=(2 / 3,1 / 3,-1)$ 
at most exponentially as $|\operatorname{Im} \gamma|,|\operatorname{Im} z| \rightarrow+\infty$. These constants will be called of admissible growth.

\section{The EASY ESTIMATES}

In this section we prove the endpoint estimates corresponding to the vertices $(1 / 2,1 / 2,-3 / 2)$ and $(1,0,0)$ of the tetrahedron. More precisely, we have the following

Proposition. (1) $S_{z}^{\gamma}$ maps $L^{2} \rightarrow L^{2}$ when $\operatorname{Re} z=-3 / 2$ and $\operatorname{Re} \gamma=0$.

(2) $S_{z}^{\gamma}$ maps $L^{1} \rightarrow L^{\infty}$ when $\operatorname{Re} z \geq 0$ and $\operatorname{Re} \gamma=3$.

In both cases the bounds are of admissible growth in $|\operatorname{Im} \gamma|,|\operatorname{Im} z| \rightarrow+\infty$.

Proof. We start by proving (2), the easier of the two estimates. Fix $\gamma$ with $\operatorname{Re} \gamma=3$ and $z$ with $\operatorname{Re} z=0$. We have

$$
\begin{aligned}
& \left|\left(S_{z}^{\gamma} f\right)(x)\right| \\
& \quad \leq 2\left|\Gamma\left(\frac{z+1}{2}\right)^{-1}\right| \iint|u-1|^{\operatorname{Re} z} \psi(u-1)\left|f\left(x_{1}-t, x_{2}-u t^{2}\right)\right| d u|t|^{\operatorname{Re} \gamma} \frac{d t}{|t|} \\
& \quad \leq C_{z} \iint\left|\frac{w}{t^{2}}-1\right|^{\operatorname{Re} z} \psi\left(\frac{w}{t^{2}}-1\right)\left|f\left(x_{1}-t, x_{2}-w\right)\right| d w d t \leq C_{z}\|f\|_{L^{1}}
\end{aligned}
$$

and this proves (2).

We continue with the proof of (1). Fix $z=-3 / 2+i \theta$ and $\gamma=i \rho$ until the end of this section. Denote by $D_{z}$ the distribution:

$$
\left\langle D_{z}, f\right\rangle=\int f(u) 2 \Gamma\left(\frac{z+1}{2}\right)^{-1}|u-1|^{z} \psi(u-1) d u .
$$

(Again $D_{z}$ is originally defined for $\operatorname{Re} z>-1$ and is analytically continued for all $z$ complex.) Let us call $K_{z}^{\gamma}$ the convolution kernel of $S_{z}^{\gamma}$. Direct calculation shows that

$$
\widehat{K_{z}^{\gamma}}\left(\xi_{1}, \xi_{2}\right)=\lim _{\substack{\varepsilon \rightarrow 0 \\ N \rightarrow+\infty}} \int_{|t|=\varepsilon}^{N} \widehat{D}_{z}\left(t^{2} \xi_{2}\right) e^{-2 \pi i t \xi_{1}}|t|^{i \rho} \frac{d t}{t} .
$$

(The limits are easily shown to exist.) We have that

$$
\begin{aligned}
\widehat{D_{z}}(v) & =2\left(\Gamma\left(\frac{z+1}{2}\right)^{-1}|u|^{z} \psi(u)\right)^{\wedge}(v) e^{-2 \pi i v} \\
& =c 2^{z} \Gamma\left(-\frac{z}{2}\right)^{-1}\left(|\cdot|^{-z-1} * \hat{\psi}\right)(v) e^{-2 \pi i v}, \quad c \neq 0,
\end{aligned}
$$

where in the last equality we used a formula on page 359 in [GS]. The behavior of $L_{z}(v)=\left(|\cdot|^{-z-1} * \hat{\psi}\right)(v)$ at $\infty$ will be of importance in the study of the Fourier transform of $K_{z}^{\gamma}$. It is easy to see that $L_{z}$ is an even $C^{\infty}$ function on the real line and, by Lemma 3.2 in [G1], we have that

$$
L_{z}(v)=c_{z}|v|^{-z-1}+O\left(|v|^{-M}\right) \quad \forall M>0 \text { as }|v| \rightarrow \infty,
$$

where all the constants above are of admissible growth and $c_{z}$ is nonzero. We will prove that $\widehat{K_{z}^{\gamma}}\left(\xi_{1}, \xi_{2}\right)$ is bounded. Fix $\xi_{2} \neq 0$, and let $\varepsilon^{\prime}=\varepsilon\left|\xi_{2}\right|^{-1 / 2}$, 
$N^{\prime}=N\left|\xi_{2}\right|^{-1 / 2}, \lambda=\xi_{1}\left|\xi_{2}\right|^{-1 / 2}$, and $\varepsilon_{2}=\operatorname{sgn} \xi_{2}$. Also let $a=c_{z, \gamma}$ be a positive large constant to be chosen later. By the evenness of $L_{z}$ we get

$$
\begin{aligned}
\widehat{K_{z}^{\gamma}}\left(\xi_{1}, \xi_{2}\right) & =\lim _{\substack{\varepsilon \rightarrow 0 \\
N \rightarrow \infty}} \int_{|t|=\varepsilon}^{N} C_{z} L_{z}\left(t^{2} \xi_{2}\right) e^{-2 \pi i\left(t \xi_{1}+\xi_{2} t^{2}\right)}|t|^{i \rho} \frac{d t}{t} \\
& =\lim _{\substack{\varepsilon^{\prime} \rightarrow 0 \\
N^{\prime} \rightarrow \infty}} \int_{|t|=\varepsilon^{\prime}}^{N^{\prime}} C_{z}\left|\xi_{2}\right|^{-i \rho / 2} L_{z}\left(t^{2}\right) e^{-2 \pi i\left(t \lambda+\varepsilon_{2} t^{2}\right)}|t|^{i \rho} \frac{d t}{t} .
\end{aligned}
$$

We now write $\widehat{K_{z}^{\gamma}}\left(\xi_{1}, \xi_{2}\right)$ as the sum of

$$
\begin{aligned}
& \lim _{\varepsilon^{\prime} \rightarrow 0} \int_{|t|=\varepsilon^{\prime}}^{a} C_{z}\left|\xi_{2}\right|^{-i \rho / 2} L_{z}\left(t^{2}\right) e^{-2 \pi i\left(t \lambda+\varepsilon_{2} t^{2}\right)}|t|^{i \rho} \frac{d t}{t}, \\
& \lim _{N^{\prime} \rightarrow \infty} \int_{|t|=a}^{N^{\prime}} C_{z}\left|\xi_{2}\right|^{-i \rho / 2} L_{z}\left(t^{2}\right) e^{-2 \pi i\left(t \lambda+\varepsilon_{2} t^{2}\right)}|t|^{i \rho} \frac{d t}{t} .
\end{aligned}
$$

Because of the smoothness of $L_{z}$ at $0,(2.1)$ remains always bounded by a constant of admissible growth for all $\lambda$ real. By the asymptotic expansion of $L_{z}$ at $\infty$, we have that

$$
\begin{aligned}
(2.2)= & \lim _{N^{\prime} \rightarrow \infty} \int_{|t|=a}^{N^{\prime}} C_{z}\left|\xi_{2}\right|^{-i \rho / 2}\left|t^{2}\right|^{-z-1} e^{-2 \pi i\left(t \lambda+\varepsilon_{2} t^{2}\right)}|t|^{i \rho} \frac{d t}{t} \\
& + \text { a remainder term that is bounded uniformly in } \lambda .
\end{aligned}
$$

The main term above is equal to

$$
C_{z}\left|\xi_{2}\right|^{-i \rho / 2} \lim _{N^{\prime} \rightarrow \infty} \int_{t=a}^{N^{\prime}} t^{i(\rho-2 \theta)} e^{-2 \pi i \varepsilon_{2} t^{2}}\left(e^{-2 \pi i t \lambda}-e^{2 \pi i t \lambda}\right) d t .
$$

The phase function $\phi(t)=-2 \pi i \varepsilon_{2}\left(t^{2} \pm t \lambda\right)+i(\rho-2 \theta) \ln t$, has second derivative $\phi^{\prime \prime}$ that satisfies $\left|\phi^{\prime \prime}(t)\right| \geq c_{z, \gamma}$ if $t \geq a$ and $a$ is large enough. Van der Corput's Lemma [Z, p. 197] now gives that the integral in (2.3) is bounded by a constant uniformly in $N^{\prime}$ and $\lambda$. Therefore $\widehat{K_{z}^{\gamma}}$ is bounded and our proposition is now proved.

\section{The MAin estimates}

So far, we have proved the estimates corresponding to the vertices $(1 / 2,1 / 2$, $-3 / 2)$ and $(1,0,0)$ of the tetrahedron. By interpolation, we get estimates for the edge in between. No strong type estimates are true for the remaining vertices, for it is known that $S_{-1}^{0}=S^{0}$ does not map $L^{1} \rightarrow L^{1}$ nor $L^{\infty} \rightarrow L^{\infty}$. Our next goal is to fill in the sides. The main result of this section is

Proposition. For $\operatorname{Re} z=-1$ and $\operatorname{Re} \gamma=3 / 2 p, S_{z}^{\gamma}$ maps $L^{p}$ to $L^{2 p}$ with bounds of admissible growth, whenever $3 / 2 \leq p<\infty$.

Proof. On the real line call $h_{z}$ the distribution $h_{z}(u)=2 \Gamma\left(\frac{z+1}{2}\right)^{-1}|u|^{z} \psi(u)$, originally defined for $\operatorname{Re} z>-1$ and extended for all $z$ by analytic continuation. Let $H_{z}$ be the distribution on $\mathbb{R}^{2}$ defined by $\delta_{x_{1}=0} h_{z}\left(x_{2}\right)$. By $\mu_{z, \gamma}$ we will denote the measure acting on functions $f$ as

$$
\left\langle\mu_{z, \gamma}, f\right\rangle=\int_{|t| \leq 1} f\left(t, t^{2}\right)|t|^{\gamma-2 z-2} \frac{d t}{t} .
$$


Fix $p, z$, and $\gamma$ as in the statement of the theorem. Let $q=2 p$. The basic property of $\mu_{z, \gamma}$ is that it convolves $L^{p}$ to $L^{q}$. This is because of Theorem 1 in [C2] that justifies the third inequality:

$$
\begin{aligned}
\left\|\mu_{z, \gamma} * f\right\|_{L^{q}} & \leq\left\|\int_{|t| \leq 1}\left|f\left(x_{1}-t, x_{2}-t^{2}\right)\right||t|^{\operatorname{Re} \gamma-1} d t\right\|_{L^{q}} \\
& \leq\left\|\int_{\mathbf{R}}\left|f\left(x_{1}-t, x_{2}-t^{2}\right)\right||t|^{\operatorname{Re} \gamma-1} d t\right\|_{L^{q}} \leq C_{p, \gamma}\|f\|_{L^{p}} .
\end{aligned}
$$

We now continue the proof of our theorem. We need to prove that

$$
\left\|\int\left\langle D_{z}(u), f\left(x_{1}-t, x_{2}-u t^{2}\right)\right\rangle|t|^{\gamma} \frac{d t}{t}\right\|_{L^{q}} \leq C_{p, z, \gamma}\|f\|_{L^{p}} .
$$

It suffices to prove that

$$
\left\|\int_{|t| \leq M}\left\langle D_{z}(u), f\left(x_{1}-t, x_{2}-u t^{2}\right)\right\rangle|t|^{\gamma} \frac{d t}{t}\right\|_{L^{q}} \leq C_{p, z, \gamma}\|f\|_{L^{p}}
$$

is valid for all $M>0$ with a bound $C_{p, z, \gamma}$ independent of $M>0$. To prove (3.1), by homogeneity we may assume that $M=1$. Let $K_{z, 1}^{\gamma}$ be the convolution kernel of the operator in (3.1) when $M=1$.

By $\chi_{A}$ we denote the characteristic function of the set $A$. We have the following

Lemma. $K_{z, 1}^{\gamma}=H_{z} * \mu_{z, \gamma}+\zeta(x)$ where $\zeta(x)$ satisfies

$$
|\zeta(x)| \leq C_{z, \gamma}\left|\frac{x_{2}}{x_{1}^{2}}-1\right|^{-1} \chi_{\left|x_{2} / x_{1}^{2}-1\right| \geq \frac{1}{2}}\left|x_{1}\right|^{\operatorname{Re} \gamma-3} .
$$

Proof. Let $\tilde{\mu}_{z, \gamma}$ denote the reflection of the measure $\mu_{z, \gamma}$ about the origin. For all Schwartz functions $g$ we have

$$
\begin{aligned}
\left\langle H_{z} * \mu_{z, \gamma}, g\right\rangle & =\left\langle H_{z}, \tilde{\mu}_{z, \gamma} * g\right\rangle \\
& =\left\langle H_{z}\left(x_{1}, x_{2}\right), \int_{|t| \leq 1} g\left(x_{1}+t, x_{2}+t^{2}\right)|t|^{\gamma-2 z-2} \frac{d t}{t}\right\rangle \\
& =\int_{|t| \leq 1} 2 \Gamma\left(\frac{z+1}{2}\right)^{-1}\left|x_{2}\right|^{z} \psi\left(x_{2}\right) \int g\left(t, x_{2}+t^{2}\right)|t|^{\gamma-2 z-2} \frac{d t}{t} d x_{2} \\
& =\int_{\left|x_{1}\right| \leq 1} \int 2 \Gamma\left(\frac{z+1}{2}\right)^{-1}\left|x_{2}-x_{1}^{2}\right|^{z} \psi\left(x_{2}-x_{1}^{2}\right) g\left(x_{1}, x_{2}\right) \\
& \times\left|x_{1}\right|^{\gamma-2 z-2} x_{1}^{-1} d x_{1} d x_{2} .
\end{aligned}
$$

It follows that $\left\langle K_{z}^{\gamma}-H_{z} * \mu_{z, \gamma}, g\right\rangle=\iint \zeta\left(x_{1}, x_{2}\right) g\left(x_{1}, x_{2}\right) d x_{1} d x_{2}$, where

$$
\zeta\left(x_{1}, x_{2}\right)=2 \Gamma\left(\frac{z+1}{2}\right)^{-1}\left|\frac{x_{2}}{x_{1}^{2}}-1\right|^{z} \frac{\left|x_{2}\right|^{\gamma-2}}{x_{1}}\left[\psi\left(\frac{x_{2}}{x_{1}^{2}}-1\right)-\psi\left(x_{2}-x_{1}^{2}\right)\right]_{\chi_{\left|x_{1}\right| \leq 1}} .
$$

Clearly $\zeta\left(x_{1}, x_{2}\right)$ satisfies the asserted estimate and this concludes the proof of the lemma.

Note that $\widehat{H}_{z}\left(\xi_{1}, \xi_{2}\right)=\hat{h}_{z}\left(\xi_{2}\right)=c_{z}\left(\left|\xi_{2}\right|^{-z-1} * \hat{\psi}\left(\xi_{2}\right)\right)$. Since $\operatorname{Re} z=-1$, the Hörmander multiplier theorem [S2, pp. 51-52] gives that convolution with $h_{z}$ 
is a bounded operator on $L^{p}(\mathbb{R})$ for $1<p<\infty$ and, therefore, convolution with $H_{z}$ is a bounded operator on $L^{p}\left(\mathbb{R}^{2}\right)$ for the same range of $p$ 's. Thus

$$
\left\|f * H_{z} * \mu_{z, \gamma}\right\|_{L^{q}} \leq C_{p, z, \gamma}\left\|f * H_{z}\right\|_{L^{p}} \leq C_{p, z, \gamma}\|f\|_{L^{p}} .
$$

It remains to control $\|f * \zeta\|_{L^{q}}$ by $C_{p, \gamma}\|f\|_{L^{p}}$. We prove that $\zeta \in L^{r, \infty}$ where $r=3 /(3-\operatorname{Re} \gamma)$. We denote by $|A|$ the Lebesgue measure of the set $A$. Let $\alpha$ be a positive number and set $\beta=\alpha^{-1 /(\operatorname{Re} \gamma-3)}$. Computation gives

$$
\begin{aligned}
|\{x:|\zeta(x)|>\alpha\}| & \leq\left|\left\{x:\left|\frac{x_{2}}{x_{1}^{2}}-1\right|^{-1} \chi_{\left|x_{2} / x_{1}^{2}-1\right| \geq 1 / 2}\left|x_{1}\right|^{\operatorname{Re} \gamma-3}>\alpha\right\}\right| \\
& =\left|\left\{x:\left|\frac{\beta^{2} x_{2}}{\left(\beta x_{1}\right)^{2}}-1\right|^{-1} \chi_{\left|\beta^{2} x_{2} /\left(\beta x_{1}\right)^{2}-1\right| \geq 1 / 2}\left|\beta x_{1}\right|^{\operatorname{Re} \gamma-3}>1\right\}\right| \\
& =\beta^{-3}\left|\left\{\left(x_{1}, x_{2}\right):\left|\frac{x_{2}}{x_{1}^{2}}-1\right|^{-1} \chi_{\left|x_{2} / x_{1}^{2}-1\right| \geq 1 / 2}\left|x_{1}\right|^{\operatorname{Re} \gamma-3}>1\right\}\right| \\
& =\alpha^{-r} m,
\end{aligned}
$$

where

$$
m=\left|\left\{\left(x_{1}, x_{2}\right):\left|\frac{x_{2}}{x_{1}^{2}}-1\right|^{-1} \chi_{\left|x_{2} / x_{1}^{2}-1\right| \geq 1 / 2}\left|x_{1}\right|^{\operatorname{Re} \gamma-3}>1\right\}\right| .
$$

We next show that $m<\infty$. This amounts to showing that the total area bounded by the following equations in $\mathbb{R}^{2}$ is finite,

$$
\frac{3}{2} x_{1}^{2} \leq x_{2} \leq x_{1}^{2}+\left|x_{1}\right|^{\operatorname{Re} \gamma-1}, \quad x_{1}^{2}-\left|x_{1}\right|^{\operatorname{Re} \gamma-1} \leq x_{2} \leq \frac{1}{2} x_{1}^{2} .
$$

This last assertion is obvious and is due to the fact that $0<\operatorname{Re} \gamma \leq 1$. We have now proved that $\zeta \in L^{r, \infty}$ where $r=3 /(3-\operatorname{Re} \gamma)$. It follows from Young's inequality that convolution with $\zeta$ maps $L^{p}$ to $L^{q}$, where $p, q$, and $r$ are related as in $1 / r+1 / p=1+1 / q$, which is equivalent to

$$
\frac{3-\operatorname{Re} \gamma}{3}+\frac{1}{p}=1+\frac{1}{q} \text { or } \operatorname{Re} \gamma=\frac{3}{2 p} \text {. }
$$

This concludes the proof of the main result of this section.

\section{CONCLUSION OF THE PROOF OF THEOREM 1}

We use the estimates of the previous sections and analytic interpolation to prove Theorem 1. We also show that this theorem describes the exact range of $p, q, z$, and $\gamma$ such that $S_{z}^{\gamma}$ maps $L^{p}$ to $L^{q}$, when $\gamma>0$. Recall that $\mathrm{A}=(0,0,-1), \mathrm{B}=(1 / 2,1 / 2,-3 / 2), \mathrm{C}=(1,1,-1)$, and $\mathrm{D}=(1,0,0)$ are the vertices of the tetrahedron, and let $E$ be the point $(2 / 3,1 / 3,-1)$. (See Figure 1.) As we mentioned before, interpolation between the points $B$ and $\mathrm{D}$ gives that on the edge $\mathrm{BD}$ our analytic family maps $L^{p}$ to $L^{p^{\prime}}$. (See Proposition in $\S 2$.) By the proposition in $\S 3$, we have strong type bounds on the closed segment $\mathrm{EC}$ minus the point $\mathrm{C}$. We now interpolate between the edges $\mathrm{BD}$ and $\mathrm{BE}-\{\mathrm{C}\}$ to get strong type bounds on the interior of the face $\mathrm{BCD}$. By duality we also fill in the interior of the face ABD. When $\gamma>0$, we have now proved strong type bounds on the interior of the bottom faces of the critical tetrahedron $A B C D$ union the point $\mathrm{D}$. Finally by interpolation we get strong type bounds for every point that lies vertically above. 
The best result known on the line segment BC is that $S_{z}^{0}$ maps $L^{p}$ to $L^{p, p^{\prime}}$, see [G1]. By duality we get that on the line segment AC, $S_{z}^{0}$ maps $L^{p^{\prime}, p}$ to $L^{p^{\prime}}$. It is easy to check that no strong type bounds hold on the open segments $\mathrm{CD}$ and $\mathrm{AD}$. However, using the fact that the analytic family $S_{z}^{\gamma}$ maps the space parabolic $H^{1}$ to weak $L^{1}$ when $\operatorname{Re} \gamma=0$ and $\operatorname{Re} z=-1$ [G1, Theorem 2], interpolation gives that on the open line segment $\mathrm{CD}, S_{z}^{\gamma}$ maps $H^{1}$ to weak $L^{p}$. Finally by duality we get that on the open line segment $\mathrm{AD}, S_{z}^{\gamma}$ maps $L^{p^{\prime}, 1}$ to parabolic BMO.

We now indicate why no boundedness results hold below the faces $B C D$ and ABD of tetrahedron. Let $\delta>0$ be small and let $f_{\delta}$ be the characteristic function of the square of sidelength $\delta$ centered at the origin. Since away from the parabola the kernel $K_{z}^{\gamma}$ looks like

$$
K_{z}^{\gamma}(x)=c_{z}\left|x_{1}\right|^{-2-2 z+\gamma} x_{1}^{-1}\left|x_{2}-x_{1}^{2}\right|^{z} \psi\left(x_{2} / x_{1}^{2}-1\right),
$$

it follows that on the set $A_{\delta}=\left\{x: x_{1} \sim 1\right.$ and $\left.\left|x_{2}-x_{1}^{2}\right| \geq 10 \delta\right\},\left|\left(S_{z}^{\gamma} f_{\delta}\right)\right|$ looks like

$$
\left|\left(S_{z}^{\gamma} f_{\delta}\right)(x)\right| \sim\left|x_{2}-x_{1}^{2}\right|^{z} \delta^{2} .
$$

Therefore,

$$
\left(\int_{A_{\delta}}\left|\left(S_{z}^{\gamma} f_{\delta}\right)(x)\right|^{q} d x\right)^{1 / q} \sim \delta^{2} \delta^{\operatorname{Re} z+1 / q},
$$

and since $\left\|f_{\delta}\right\|_{L^{p}}=\delta^{2 / p}$, letting $\delta \rightarrow 0$ and comparing exponents, we see that no inequality of the form $\left\|S_{z}^{\gamma} f\right\|_{L^{q}} \leq C\|f\|_{L^{p}}$ is possible when $1 / q<$ $2 / p-2-\operatorname{Re} z$. Note that for a fixed $z, 1 / q=2 / p-2-\operatorname{Re} z$ is the equation of the line that intersects the segments $B D$ and $C D$ and is parallel to the line $C E$ at height $(0,0, \operatorname{Re} z)$. By duality we get that boundedness cannot hold when $2 / q<1 / p-1-\operatorname{Re} z$. Again for a fixed $z, 2 / q=1 / p-1-\operatorname{Re} z$ is the equation of the line that intersects the segments $\mathrm{BD}$ and $\mathrm{AD}$ and is parallel to the line $\mathrm{AE}$ at height $(0,0, \operatorname{Re} z)$. We have now proved that for a fixed $z, L^{p} \rightarrow$ $L^{q}$ boundedness cannot hold when the point $(1 / p, 1 / q, \operatorname{Re} z)$ lies outside the triangle with vertices $A^{\prime} E^{\prime} C^{\prime}$ where $A^{\prime}, E^{\prime}$, and $C^{\prime}$ are the intersections of the lines $\mathrm{BA}, \mathrm{BE}$, and $\mathrm{BC}$ with the horizontal plane through $(0,0, \operatorname{Re} z)$. This intersection is interesting to us only when $-3 / 2 \leq \operatorname{Re} z \leq-1$. The same argument applies to the degenerate case when the triangle $\mathrm{A}^{\prime} \mathrm{E}^{\prime} \mathrm{C}^{\prime}$ becomes the point $B$.

\section{THE HeISENBERG GROUP PROBLEM}

In this section we discuss a similar issue on the Heisenberg group $\mathbb{H}^{n}$. $\mathbb{H}^{n}$ is the Lie group with underlying manifold $\mathbb{C}^{n} \times \mathbb{R}$ and with multiplication law $(z, t)\left(z^{\prime}, t^{\prime}\right)=\left(z+z^{\prime}, t+t^{\prime}+2 \operatorname{Im} z \cdot \bar{z}^{\prime}\right)$ where $z \cdot \bar{z}^{\prime}=\sum_{j=1}^{n} z_{j} \bar{z}_{j}^{\prime}$. The norm of an element $u=(z, t) \in \mathbb{H}^{n}$ is defined by $|u|=\left(|z|^{4}+|t|^{2}\right)^{1 / 4}$ and is homogeneous of degree 1 under the one-parameter group of dilations $r(z, t) \rightarrow\left(r z, r^{2} t\right)$. Let $\delta$ be the Dirac distribution in the $t$ variable. Ricci and Stein [RS] considered the family of operators

$$
S^{\gamma} f=f *\left[\Gamma\left(\frac{\gamma+1}{2}\right)^{-1}|z|^{\gamma-2 n} \delta_{t=0}\right]
$$


for $0<\gamma \leq 2 n$, where $*$ is the Heisenberg group convolution. Define $\Gamma$ to be the closed triangle in $\mathbb{R}^{2}$ with vertices $(0,0),(1,1)$, and $\left(1 / p_{0}, 1 / q_{0}\right)$ minus the diagonal $\{(p, q): 1 / p=1 / q\}$, where

$$
p_{0}=1+(2 n+1)^{-1}, \quad q_{0}=2 n+2 .
$$

When $n=1$, Ricci and Stein obtained $L^{p} \rightarrow L^{q}$ boundedness of $S^{\gamma}$ for $(1 / p, 1 / q)$ in the interior of $\Gamma$ and on a portion of its boundary, namely, when $6 / 5 \leq p \leq 2$. Christ [C2] proved $L^{p} \rightarrow L^{q}$ boundedness for all boundary points of $\Gamma$ that do not lie on the diagonal for all $n \geq 1$. Furthermore, an example given in [C2] shows that no boundedness result can hold outside the closure of $\Gamma$. (The singular integral case $\gamma=0$ has been treated by Geller and Stein [GSt].)

In this section we prove a similar result as in [C2] for an analytic family of fractional integrals $S_{w}^{\gamma}$, in which the operators $S^{\gamma}$ can be embedded. The kernels of $S_{w}^{\gamma}$ are obtained by taking $-w-1$ derivatives transverse to $\mathbb{C}^{n}$, and doing so in a dilation invariant way. Again the analytic family is defined in such a way as to satisfy $S_{-1}^{\gamma}=S^{\gamma}$. Fix a real smooth even nonnegative compactly supported bump function $\psi$ equal to 1 in a neighborhood of 0 . Our analytic family $S_{w}^{\gamma}$ is given by convolution with the distribution

$$
K_{w}^{\gamma}(z, t)=|z|^{\gamma-2 n-2 w-2} \Gamma\left(\frac{w+1}{2}\right)^{-1}|t|^{w} \psi\left(t /|z|^{2}\right) .
$$

For $\operatorname{Re} w>-1$, one can define $S_{w}^{\gamma}$ as

$$
\iint f\left(z-z^{\prime}, t-u\left|z^{\prime}\right|^{2}-2 \operatorname{Im} z \cdot z^{\prime}\right)|u|^{w} \psi(u) d u\left|z^{\prime}\right|^{\gamma-2 n} d z^{\prime} .
$$

By analytic continuation, $S_{w}^{\gamma}$ can be defined to be a distribution-valued entire function of $w$ with the property $S_{-1}^{\gamma}=S^{\gamma}$. Our second result describes the exact range of $(1 / p, 1 / q, z, \gamma)$ for which $S_{z}^{\gamma}$ maps $L^{p}\left(\mathbb{H}^{n}\right)$ to $L^{q}\left(\mathbb{H}^{n}\right)$ when $\operatorname{Re} \gamma>0$. Since by homogeneity considerations, such a boundedness result can only hold when $1 / p-1 / q=\operatorname{Re} \gamma / 2(n+1)$, it is enough to describe the possible range of $p, q$, and $w$ for which $S_{w}^{\gamma}$ maps $L^{p}$ to $L^{q}$. The precise statement of the theorem is

Theorem 2. For $\operatorname{Re} \gamma>0$, the analytic family of fractional integrals $S_{w}^{\gamma}$ maps $L^{p}$ to $L^{q}$ if and only if $(1 / p, 1 / q, \operatorname{Re} w)$ lies on or vertically above the interiors of the faces $\mathrm{BCD}$ and $\mathrm{ABD}$ union the segment $\mathrm{BD}-\{\mathrm{B}\}$ of the tetrahedron $\mathrm{ABCD}$ with vertices $\mathrm{A}=(0,0,-1), \mathrm{B}=(1 / 2,1 / 2,-n-1), \mathrm{C}=(1,1,-1)$, and $\mathrm{D}=(1,0,0) .($ See Figure 2.$)$

Proof. Again, we will use Theorem 2 in [C2] to treat part of the kernel of $S_{w}^{\gamma}$. The proof of this theorem is similar to the proof of Theorem 1. The $L^{2}$ boundedness follows from the work of Geller and Stein [GSt]. They prove that if $\Phi(z, t) \in C^{\infty}\left(\mathbb{H}^{n}-\{0\}\right)$, homogeneous of degree $0,0 \leq \Phi \leq 1$ and such that for some $C_{0}>0, \Phi(z, t)=1$ if $|t| \leq C_{0}|z|^{2}, \Phi(z, t)=0$ if $|t| \geq C_{0}|z|^{2}$, then $\mathbb{H}^{n}$-convolution with the distribution $\Gamma\left(\gamma^{\prime} / 2\right)^{-1} \Phi(z, t)|z|^{-2\left(n+\gamma^{\prime}\right)}|t|^{-1+\gamma^{\prime}}$ maps $L^{2}$ to $L^{2}$ with bounds of admissible growth if and only if $\operatorname{Re} \gamma^{\prime} \geq-n$. (In their paper $\gamma^{\prime}$ is denoted by $\gamma$.) Setting $\gamma^{\prime}=w+1$ and $\Phi(z, t)=\psi\left(t /|z|^{2}\right)$, we get that when $\operatorname{Re} \gamma=0, S_{w}^{\gamma}$ maps $L^{1}$ to $L^{2}$ if and only if $\operatorname{Re} w \geq-(n+1)$. 


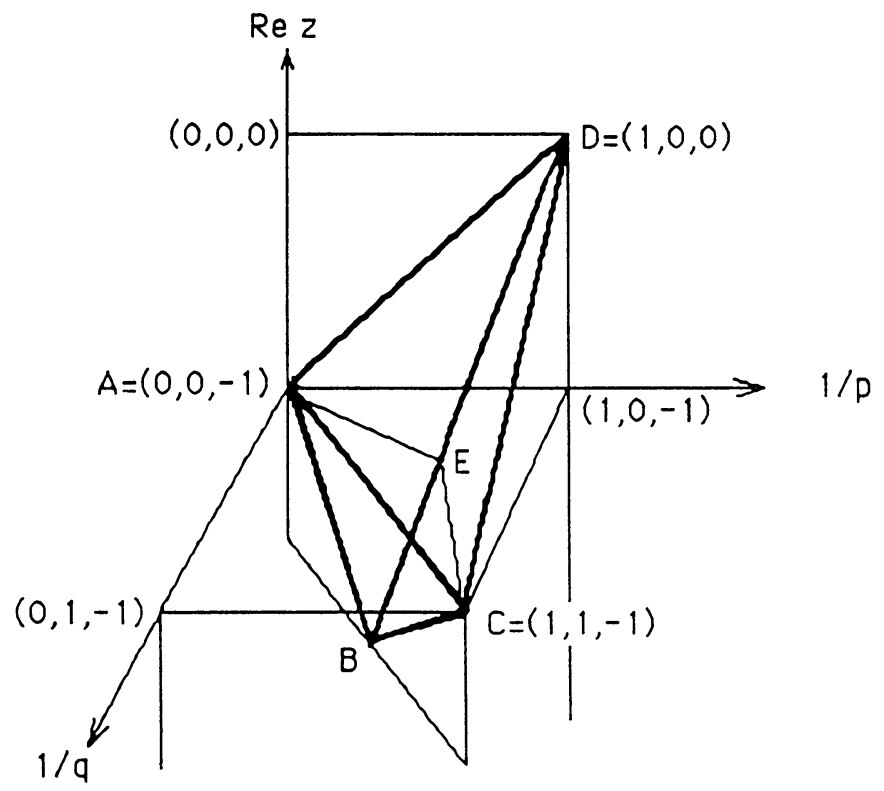

FigURE 2. $\mathrm{B}=(2 n+1 / 2(n+1), 1 / 2(n+1),-1), \mathrm{E}=$ $(1 / 2,1 / 2,-n-1)$

Also, one can easily see that when $\operatorname{Re} \gamma=2(n+1), S_{w}^{\gamma}$ maps $L^{1}$ to $L^{\infty}$ if and only if $\operatorname{Re} w \geq 0$. Analytic interpolation gives that for $(1 / p, 1 / q, \operatorname{Re} w) \in \mathrm{BD}$, $S_{w}^{\gamma}$ maps $L^{p}$ to $L^{q}$. (Here $q=p^{\prime}$.)

Let $\mathrm{E}=\left(1 / p_{0}, 1 / q_{0},-1\right)$. Our proof will be complete by interpolation if we can show that for $(1 / p, 1 / q,-1)$ in the segment $\mathrm{AE}-\{\mathrm{A}\}$ and $\operatorname{Re} w=-1$, $S_{w}^{\gamma}$ maps $L^{p}$ to $L^{q}$ with bounds of admissible growth. To prove this, let us fix $p, q, \gamma$, and $w$ with $\operatorname{Re} w=-1$, set $K_{w, 1}^{\gamma}=K_{w}^{\gamma} \chi_{|z| \leq 1}$, and define a distribution $H_{w}=\Gamma\left(\frac{w+1}{2}\right)^{-1}|t|^{w} \psi(t) \delta_{z=0}$. By $k_{w, \gamma}$ we will denote the kernel $\delta_{t=0}|z|^{\gamma-2 n-2 w-2} \chi_{|z| \leq 1}$. The basic property of $k_{w, \gamma}$ is that it convolves $L^{p}$ to $L^{q}$. This is because of the following inequalities:

$$
\begin{gathered}
\left\|f * k_{w, \gamma}\right\|_{L^{q}}=\left\|\int_{\left|z^{\prime}\right| \leq 1}\left|f\left(z-z^{\prime}, t-2 \operatorname{Im} z \cdot \bar{z}^{\prime}\right)\right|\left|z^{\prime}\right|^{\gamma-2 n-2 w-2} d z^{\prime}\right\|_{L^{q}}, \\
\left\|\int_{\mathbb{C}^{n}}\left|f\left(z-z^{\prime}, t-2 \operatorname{Im} z \cdot \bar{z}^{\prime}\right)\right|\left|z^{\prime}\right|^{\gamma-2 n} d z^{\prime}\right\|_{L^{q}} \leq C_{p, \gamma}\|f\|_{L^{p}} .
\end{gathered}
$$

The last inequality follows from Theorem 2 in [C2]. We need the following

Lemma. $K_{w, 1}^{\gamma}=k_{w, \gamma} * H_{w}+\zeta(z, t)$ where $\zeta(z, t)$ satisfies

$$
|\zeta(z, t)| \leq C_{w} \chi_{|t| \geq|z|^{2}}|z|^{\operatorname{Re} \gamma-2 n-2}\left(|t| /|z|^{2}\right)^{-1} .
$$

Proof. The proof of the lemma follows from an easy calculation. We have that

$$
\begin{aligned}
& \left(f * k_{w, \gamma} * H_{w}\right)(z, t) \\
& \quad=\int_{\left|z^{\prime}\right| \leq 1} \int f\left(z-z^{\prime}, t-t^{\prime}-2 \operatorname{Im} z \cdot \bar{z}^{\prime}\right)\left|z^{\prime}\right|^{\gamma-2 n-2 w-2} \frac{\left|t^{\prime}\right|^{w}}{\Gamma\left(\frac{w+1}{2}\right)} \psi\left(t^{\prime}\right) d t^{\prime} d z^{\prime} .
\end{aligned}
$$


It follows that the difference $f * K_{w, 1}^{\gamma}-f * k_{w, \gamma} * H_{w}$ is equal to $f * \zeta$, where

$$
\zeta(z, t)=\Gamma\left(\frac{w+1}{2}\right)^{-1}|z|^{\gamma-2 n-2 w-2}|t|^{w}\left[\psi\left(\frac{t}{|z|^{2}}\right)-\psi(t)\right] \chi_{|z| \leq 1}
$$

Since $\psi$ vanishes near 0 , the function $\zeta$ above is supported in $|t| \geq|z|^{2}$ and the required estimate for $\zeta$ follows.

To prove the theorem, it is enough to consider $K_{w, M}^{\gamma}=K_{w}^{\gamma} \chi_{|z| \leq M}$ for all $M>0$ and prove that they convolve $L^{p}$ to $L^{q}$ with bounds uniform in $M$. By homogeneity, it suffices to prove that $K_{w, 1}^{\gamma}$ convolves $L^{p}$ to $L^{q}$. This will be a consequence of the lemma. First note that for all $1<q<\infty$, $\left\|f * H_{w}\right\|_{L^{q}} \leq C_{w, q}\|f\|_{L^{q}}$ is a consequence of the Hörmander multiplier theorem. It then follows that

$$
\left\|\left(f * k_{w, \gamma}\right) * H_{w}\right\|_{L^{q}} \leq C_{q, w}\left\|f * k_{w, \gamma}\right\|_{L^{q}} \leq C_{p, w, \gamma}\|f\|_{L^{p}} .
$$

It remains to control $\|f * \zeta\|_{L^{q}}$ by $C_{p, w, \gamma}\|f\|_{L^{p}}$. Similar argument as in $\S 3$ gives that $\zeta \in L^{r, \infty}$ where $r=(2 n+2) /(2 n+2-\operatorname{Re} \gamma)$. Young's inequality gives that convolution with $\zeta$ maps $L^{p}$ to $L^{q}$, where $p, q$, and $r$ are related as in $1 / r+1 / p=1+1 / q$, which is equivalent to $1 / p-1 / q=\operatorname{Re} \gamma / 2(n+1)$. This concludes the proof of our claim. The proof of Theorem 2 follows from interpolation. The fact that Theorem 2 describes the exact range of $p, q, w$, and $\gamma$ for which $S_{w}^{\gamma}$ maps $L^{p}$ to $L^{q}$ when $\gamma>0$, follows from an argument similar to the one given in $\S 4$. We omit the details.

\section{ACKNOWLEDGMENT}

This paper is a natural continuation of the work I did in my thesis and I would like to thank my advisor, Mike Christ, once again.

\section{REFERENCES}

[BL] J. Bergh and J. Löfström, Interpolation spaces, an introduction, Springer Verlag, Berlin, Heidelberg, and New York, 1976.

[C1] M. Christ, Weak type $(1,1)$ bounds for rough operators, Ann. of Math. (2) 128 (1988), 19-42.

[C2] _ Endpoint bounds for singular fractional integral operators, preprint.

[GS] I. M. Gelfand and G. E. Shilov, Generalized functions, Vol. I, Academic Press, New York and London, 1964.

[GSt] D. Geller and E. M. Stein, Estimates for singular convolution operators on the Heisenberg group, Math. Ann. 267 (1984), 1-15.

[G1] L. Grafakos, Endpoint bounds for an analytic family of Hilbert transforms, Duke Math. J. 62 (1991), 23-59.

[G2] _- Weak type estimates for a singular convolution operator on the Heisenberg group, Trans. Amer. Math. Soc. 325 (1991), 435-452.

[NSW] A. Nagel, E. M. Stein, and S. Wainger, Differentiation in lacunary directions, Proc. Nat. Acad. Sci. U.S.A. 75 (1978), 1060-1062.

[O] D. Oberlin, Convolution estimates for some measures on curves, Proc. Amer. Math. Soc. 99 (1987), 56-60.

[RS] F. Ricci and E. M. Stein, Harmonic analysis on nilpotent groups and singular integrals III: Fractional integration along manifolds, MSRI preprint, 1988. 
[S1] E. M. Stein, Problems in harmonic analysis related to curvature and oscillatory integrals, Proceedings of the International Congress of Mathematicians, Berkeley, CA, 1986.

[S2] _ Singular integrals and differentiability properties, Princeton Univ. Press, Princeton, NJ, 1970.

[SWA] E. M. Stein and S. Wainger, Problems in harmonic analysis related to curvature, Bull. Amer. Math. Soc. 84 (1978), 1239-1295.

[Z] A. Zygmund, Trigonometric series, Vol. I, 2nd ed., Cambridge Univ. Press, London, 1959.

Department of Mathematics, Yale University, New Haven, Connecticut 06520

Current address: Department of Mathematics, Washington University in St. Louis, Campus Box 1146, 1 Brookings Drive, St. Louis, Missouri 63130-4899

E-mail address: grafakos@loml.math.yale.edu 